\title{
Terlipressin Induced Severe Hyponatremia
}

\author{
Martin Šíma', Miroslav Pokorný2 ${ }^{1}$, František Pad’our ${ }^{2}$, Ondřej Slanař ${ }^{1}$ \\ ${ }^{1}$ Institute of Pharmacology, First Faculty of Medicine, Charles University in Prague \\ and General University Hospital in Prague, Prague, Czech Republic; \\ ${ }^{2} 1^{\text {st }}$ Department of Internal Medicine, Na Bulovce Hospital, Prague, Czech Republic
}

Received June 29, 2015; Accepted February 24, 2016.

Key words: Terlipressin - Hyponatremia - Adverse drug reaction - Vasopressin receptor - Fluid balance

Abstract: Terlipressin is a vasopressin analogue used for its vasoconstrictor effect in the treatment of variceal bleeding. Despite its good safety profile compared to vasopressin, some adverse reactions may occur during its use e.g. hyponatremia. We describe a case of a cirrhotic patient with active variceal bleeding treated during two separate hospitalizations with terlipressin. In both drug treatment periods, severe laboratory hyponatremia developed. After terlipressin discontinuation, mineral disbalance corrected rapidly. Positive dechallenge and rechallenge corresponding to the drug administration schedule confirms the causality between terlipressin administration and hyponatremia. Hyponatremia was preceded with substantial fluid retention in both episodes. In this case report we want to highlight the need for fluid balance monitoring immediately after first terlipressin dose, which may individually predict the patient risk for the development of hyponatremia as other risk factors have rather limited predictive value in real clinical settings.

This study was supported by grant SVV-2015-260 155.

Mailing Address: Martin Šíma, PharmDr., Institute of Pharmacology, First Faculty of Medicine, Charles University in Prague and General University Hospital in Prague, Albertov 4, 12800 Prague 2, Czech Republic; Phone: +420 224964 135; e-mail: sima.mart@seznam.cz 


\section{Introduction}

Terlipressin (1-triglycil-8-lysine-vasopressin) is a synthetic analogue of natural hormone vasopressin with long half-life and relatively low incidence of adverse effects (Freeman et al., 1982). It is frequently used in the treatment of gastrointestinal bleeding in patients with oesophageal and gastric varices, since it is a potent vasoconstrictor in the splanchnic circulation (Garcia-Tsao et al., 2007). Hyponatremia is a known adverse effect with estimated incidence of 0.1 to $1 \%$ treated patients according to the originator's summary of product characteristics. However, some publications estimate the incidence of this adverse effect higher in the real life use of the drug. Escorsell et al. (2000) report four cases of hyponatremia per 105 patients treated with terlipressin (3.81\%), while Feu et al. (1996) report five cases of hyponatremia per 80 patients treated with terlipressin (6.25\%).

\section{Case report}

A 34-year-old woman $(60 \mathrm{~kg}, 165 \mathrm{~cm})$ presented to the Department of Internal Medicine with hematemesis. The patient was known to have been diagnosed with oesophageal varices, Mallory-Weiss syndrome, portal hypertension and liver cirrhosis (Child-Pugh B) - most probably of ethylic etiology. When admitting, her blood pressure was $90 / 55 \mathrm{~mm} \mathrm{Hg}$, heart rate 70 beats/min, respiratory rate 14 breaths/min, and blood oxygen level $99 \%$. Her initial laboratory findings were as follows: glucose $7.80 \mathrm{mmol} / \mathrm{l}$, sodium $134.00 \mathrm{mmol} / \mathrm{l}$, potassium $4.32 \mathrm{mmol} / \mathrm{l}$, chloride $98.00 \mathrm{mmol} / \mathrm{l}$, urea $5.84 \mathrm{mmol} / \mathrm{l}$, creatinine $66.00 \mu \mathrm{mol} / \mathrm{l}$, total bilirubin $47.00 \mu \mathrm{mol} / \mathrm{l}$, conjugated bilirubin $17.00 \mu \mathrm{mol} / \mathrm{l}$, aspartate aminotransferase $46.47 \mathrm{IU} / \mathrm{l}$, alanine aminotransferase $13.52 \mathrm{IU} / \mathrm{l}$, alcaline phosphatase $162.94 \mathrm{IU} / \mathrm{l}$, gamma-glutamyl transferase $377.65 \mathrm{IU} / \mathrm{l}$, alpha amylase $40.00 \mathrm{IU} / \mathrm{l}$, C-reactive protein $34.10 \mathrm{mg} / \mathrm{l}$, prothrombin time $17.50 \mathrm{~s}$ (international normalized ratio 1.43). Acute gastroscopy confirmed bleeding varices in the oesophagus and in the cardia. Bleeding was stopped by ligation rings deployment, the patient was placed in the intensive care unit and terlipressin therapy was started $(1 \mathrm{mg}$ at $22 \mathrm{~h})$. The next two days terlipressin was administered at a dose of $1 \mathrm{mg}$ every 4 hours, followed $1 \mathrm{mg}$ dose every 6 hours for the next two days, further reduced to $1 \mathrm{mg}$ every 8 hours for the next day. Severe hyponatremia with rapid onset began to develop on day 2 of terlipressin dosing (Figure 1). Sodium levels returned to physiological range also relatively rapidly within 3 days after treatment discontinuation. While fluid intake was stable of approximately $4,000 \mathrm{ml}$ per day, fluid output was $1,650-1,900 \mathrm{ml}$ for the period of full terlipressin therapy, in the course of discontinuation it gradually increased and after complete discontinuation it reached 8,050 ml.Variceal bleeding was stopped and after internal condition adjusting, the patient was moved to a standard care unit, from where she was discharged after three days.

Fifteen months later, this female patient was hospitalized for the same diagnosis again. Her status was: Child-Pugh B, blood pressure $110 / 80 \mathrm{~mm} \mathrm{Hg}$, heart rate 


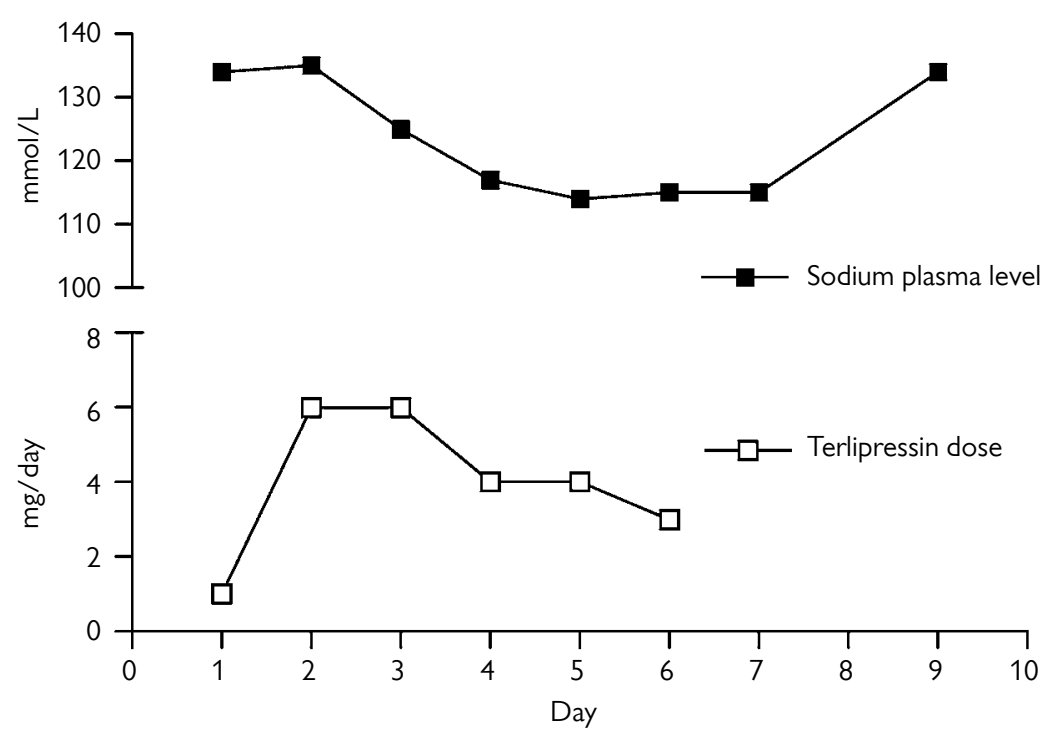

Figure 1 - Terlipressin dosing and plasma sodium levels during hospitalization.

110 beats/min, respiratory rate 14 breaths/min, and blood oxygen level $95 \%$. Initial laboratory findings were: glucose $8.90 \mathrm{mmol} / \mathrm{l}$, sodium $137.00 \mathrm{mmol} / \mathrm{l}$, potassium $3.76 \mathrm{mmol} / \mathrm{l}$, chloride $96.00 \mathrm{mmol} / \mathrm{l}$, urea $8.04 \mathrm{mmol} / \mathrm{l}$, creatinine $67.00 \mu \mathrm{mol} / \mathrm{l}$, total bilirubin $69.00 \mu \mathrm{mol} / \mathrm{l}$, conjugated bilirubin $44.00 \mu \mathrm{mol} / \mathrm{l}$, aspartate aminotransferase $110.59 \mathrm{IU} / \mathrm{l}$, alanine aminotransferase $22.94 \mathrm{IU} / \mathrm{l}$, alcaline phosphatase $201.18 \mathrm{IU} / \mathrm{l}$, gamma-glutamyl transferase $1325.88 \mathrm{IU} / \mathrm{I}$, alpha amylase $21.76 \mathrm{IU} / \mathrm{l}$, C-reactive protein $15.5 \mathrm{mg} / \mathrm{l}$, prothrombin time $17.4 \mathrm{~s}$ (international normalized ratio 1.44). She was treated with terlipressin under the same schedule as in the first case. Severe hyponatremia developed rapidly again, however, minimum sodium level was numerically higher than in the first course of the treatment $(126 \mathrm{mmol} / \mathrm{l})$. Management of laboratory hyponatremia that was not accompanied with clinical symptoms in either of the two occasions included careful clinical monitoring and hypertonic saline administration.

\section{Discussion}

Three vasopressin receptor subtypes have been identified: $\mathrm{V}_{1}$ (also known as $\mathrm{V}_{1 \mathrm{a}}$ ), $\mathrm{V}_{2}$ and $\mathrm{V}_{3}$ (also known as $\mathrm{V}_{1 \mathrm{~b}}$ ). $\mathrm{V}_{1}$ receptors are mainly located in smooth muscle cells of blood vessels, signal transduction is mediated by phospholipase $C$ activation and release of intracellular calcium, and their activation leads to vasoconstriction. $V_{2}$ receptors are mainly located in renal collecting duct, signal transduction is mediated by adenylyl cyclase activation and increase in CAMP, and their activation leads to expression of aquaporins and their 
incorporation into the membranes resulting in water reabsorption increase. $V_{3}$ receptors are mainly located in pituitary gland, signal transduction is mediated by adenylyl cyclase activation and increase in cAMP, and their activation leads to ACTH secretion increase (Saner et al., 2007; Krag et al., 2011). Terlipressin was developed as a selective $V_{1}$ receptor agonist. However, terlipressin has also substantial affinity to the $\mathrm{V}_{2}$ receptors (Krag et al., 2008). As a result of $\mathrm{V}_{2}$ receptors activation, water reabsorption in the renal collecting duct increases and dilutional hyponatremia may develop. This effect has been twice observed in our patient with diuresis reaching only less than half of the daily fluid intake during terlipressin administration and compensatory fluid overexcretion after terlipressin discontinuation. Since we observed severe hyponatremia on two separate treatment occasions with positive dechallenge (an adverse event which disappears on withdrawal of the medication) and rechallenge (symptoms re-occurring on re-administration) the causality between terlipressin administration and hyponatremia may be considered confirmed in our case. Kang et al. (2013) and Yim et al. (2015) identified that higher initial sodium plasma levels, lower patients' age, and preserved hepatic functions represent independent risk factors for development of hyponatremia. Our patient characteristics fit into this stratification, predicting the patients of increased risk for terlipressin-induced hyponatremia. However, the predictive value of these factors in reality is rather limited.

Of clinical importance is our observation that substantial fluid retention was noted within the first 24 hours after the first dose of terlipressin, while sodium level decline was first noted 32 hours after the first dose. Therefore for the practical terlipressin management, careful fluid balance monitoring immediately after the treatment initiation may be helpful for individual prediction of hyponatremia. Since the monitoring of fluid balance is a simple, non-invasive and very cheap technique not requiring any specific equipment or repeated blood samplings, it should be widely introduced into clinical monitoring of terlipressin treated patients for individual risk prediction.

Too rapid correction of serum sodium levels can cause serious neurological sequelae. Therefore plasma sodium correction must be realized carefully with frequent monitoring (Sterns, 2015). If the hyponatremia develops in the terlipressin therapy, it is also possible to consider the gradual discontinuation of drug, as in the described case reports.

\section{Conclusion}

Even though terlipressin-induced hyponatremia is a rare adverse reaction, it should always be taken into account when using this drug. Monitoring of fluid balance/ retention shortly after the first dose of the treatment may be predictive for development of severe hyponatremia and should be introduced into the clinical terlipressin management monitoring scheme. 


\section{References}

Escorsell, A., Ruiz del Arbol, L., Planas, R., Albillos, A., Bañares, R., Calès, P., Pateron, D., Bernard, B., Vinel, J. P., Bosch, J. (2000) Multicenter randomized controlled trial of terlipressin versus sclerotherapy in the treatment of acute variceal bleeding: the TEST study. Hepatology 32(3), 471-476.

Feu, F., Ruiz del Arbol, L., Bañares, R., Planas, R., Bosch, J. (1996) Double-blind randomized controlled trial comparing terlipressin and somatostatin for acute variceal hemorrhage. Variceal Bleeding Study Group. Gastroenterology 111(5), 1291-1299.

Freeman, J. G., Cobden, I., Lishman, A. H., Record, C. O. (1982) Controlled trial of terlipressin (“Glypressin”) versus vasopressin in the early treatment of oesophageal varices. Lancet 2(8289), 66-68.

Garcia-Tsao, G., Sanyal, A. J., Grace, N. D., Carey, W.; Practice Guidelines Committee of the American Association for the Study of Liver Diseases; Practice Parameters Committee of the American College of Gastroenterology (2007) Prevention and management of gastroesophageal varices and variceal hemorrhage in cirrhosis. Hepatology 46(3), 922-938.

Kang, Y. J., Bae, E. J., Hwang, K., Jeon, D. H., Jang, H. N., Cho, H. S., Chang, S. H., Park, D. J. (2013) Initial serum sodium concentration determines the decrease in sodium level after terlipressin administration in patients with liver cirrhosis. Springerplus 2, 519.

Krag, A., Bendtsen, F., Pedersen, E. B., Holstein-Rathlou, N. H., Møller, S. (2008) Effects of terlipressin on the aquaretic system: evidence of antidiuretic effects. Am. J. Physiol. Renal Physiol. 295(5), F1295-F1300.

Krag, A., Pedersen, E. B., Møller, S., Bendtsen, F. (2011) Effects of the vasopressin agonist terlipressin on plasma CAMP and ENaC excretion in the urine in patients with cirrhosis and water retention. Scand. J. Clin. Lab. Invest. 71(2), 112-116.

Saner, F. H., Canbay, A., Gerken, G., Broelsch, C. E. (2007) Pharmacology, clinical efficacy and safety of terlipressin in esophageal varices bleeding, septic shock and hepatorenal syndrome. Expert Rev. Gastroenterol. Hepatol. 1(2), 207-217.

Sterns, R. H. (2015) Disorders of plasma sodium - Causes, consequences, and correction. N. Engl. J. Med. 372(1), 55-65.

Yim, S.Y., Seo, Y. S., Jung, C. H., Kim, T. H., Kim, E. S., Keum, B., Kim, J. H., An, H., Yim, H. J., Yeon, J. E., Jeen, Y.T., Lee, H. S., Chun, H. J., Byun, K. S., Um, S. H., Kim, C. D., Ryu, H. S. (2015) Risk factors for developing hyponatremia during terlipressin treatment: a retrospective analyses in variceal bleeding. J. Clin. Gastroenterol. 49(7), 607-612. 